\title{
Carrier Frequency Offset in Coordinated Multibase MIMO Narrowband Systems
}

\author{
Brad Zarikoff*, James K. Cavers ${ }^{\dagger}$ \\ School of Engineering Science, Faculty of Applied Sciences \\ Simon Fraser University \\ Burnaby, BC, Canada V5A 1S6 \\ \{*bwzariko@sfu.ca, ${ }^{\dagger}$ cavers@sfu.ca\}
}

\begin{abstract}
The effect of carrier frequency offset (CFO) in a coordinated multibase MIMO downlink system is considered. We begin with evidence that uncompensated base station (BS) CFOs can significantly reduce the achievable capacity of such systems. Correction of the CFOs is desirable, and a critical step in doing so is estimation of their values. We present a method by which mobile subscribers (MSs) can estimate multiple CFOs from training sequences received from the BSs. Compared to existing estimators, the proposed estimator gives better accuracy with a lower transmit power.
\end{abstract}

\section{INTRODUCTION}

$\mathbf{C}$ OORDINATED multibase MIMO systems, as proposed in [1],[2],[3], share information in order to create a single, very large MIMO system. The information exchange between the basestations includes data, complex channel gains, and queuing information. Such coordinated systems have a number of technical challenges to overcome before practical implementation. For example, if the downlink of such a system relies on spatial zero forcing (ZF) to separate signals to different users, degraded CSI can cause some loss of orthogonality, and therefore increased interference and reduced capacity. For typical BS frequency accuracies (e.g., $\varepsilon_{0}=1 \mathrm{ppm}$ ), it is the carrier frequency offsets (CFOs), more than the Dopplerinduced instability of the medium, that cause the deterioration of CSI with time. In this work, the effect of independent CFOs at each BS will be briefly examined in the context of a flatfading, symbol synchronous channel.

Ultimately, CFOs should be corrected. As a first step towards this goal, they must be estimated. Therefore, our second topic in this paper is a method for estimation of the several CFOs by each mobile subscriber (MS). Unlike existing methods [4],[5],[6] our method copes with loss of orthogonality of training sequences and requires much less peak power from the expensive radio power amplifiers.

\section{BACKGROUND}

\section{A. System Model}

The following system model, shown in Fig. 1, is intentionally simplistic in order to keep the effects of CFO easily

This work was supported by a National Sciences and Engineering Research Council (NSERC) of Canada Post Graduate Scholarship. Submitted to IEEE VTC Spring 2008. recognizable and to demonstrate the effects on coordinated systems.

For a time and frequency invariant channel, the received signal is downconverted to baseband and matched filtered. The output of the matched filter contains sufficient statistics at one sample per symbol, given that the CFO effect over the signaling pulse duration is negligible. An orthogonal multiuser technique is used by the BSs to prevent interference between transmitted streams. However, BS CFOs cause phase errors to accumulate in the time between MS channel estimation and receipt of those estimates at the BSs, as well as during the transmission. The phase errors degrade orthogonality, causing increased interference and reduced capacity.

For $B$ BSs and $K$ MSs, each with $N_{T}$ and $N_{R}$ antennas respectively, the model is shown in Fig. $1 . \mathbf{H}_{k, b}$ is the $N_{R} \times N_{T}$ matrix of channel gains from the $b^{\text {th }} \mathrm{BS}$ to the $k^{\text {th }} \mathrm{MS}$, which we consider to be static over the estimation period. The CFO is considered as the frequency offset of the LO from an ideal carrier frequency. Of course the MS has a CFO of its own, and any estimates of BS CFO are relative to the MS CFO. For the purposes of this paper, however, it is simplest to assume that the MS CFO is zero. For the $k^{t h}$ MS, the vector of received samples from the $N_{R}$ antennas at the $n^{t h}$ symbol interval is

$$
\mathbf{r}_{k}(n)=\sum_{i=1}^{K} \mathbf{H}_{k} \mathbf{D}(\mathbf{c} n) \mathbf{x}_{i}(n)+\mathbf{n}_{k}(n)
$$

The length- $N_{R}$ complex vector $\mathbf{r}_{k}(n)$ contains the received samples at the $k^{t h}$ MS, the $N_{R} \times B N_{T}$ complex matrix $\mathbf{H}_{k}=$ $\left[\mathbf{H}_{k, 1}, \cdots, \mathbf{H}_{k, B}\right]$ contains all channel gains for the $k^{t h} \mathrm{MS}$, the $B N_{T} \times B N_{T}$ complex diagonal matrix $\mathbf{D}(\mathbf{c} n)$ contains the unwanted phase offsets of the $B$ BSs with the length- $B$ vector $\mathbf{c}$ containing the CFOs normalized by the symbol rate (detailed in the following section), the length- $B N_{T}$ complex vector $\mathbf{x}_{i}(n)$ contains the transmitted signal from the $B$ BSs to the $i^{\text {th }}$ MS, and the length- $N_{R}$ complex vector $\mathbf{n}_{k}(n)$ contains the additive white Gaussian noise at the $k^{\text {th }}$ MS. The $\mathbf{x}_{i}(n)$ satisfy $\sum_{i=1}^{K} \mathbf{x}_{i}^{\dagger}(n) \mathbf{x}_{i}(n)=E_{B S}$, where $E_{B S}$ is the total transmit energy for all MS and BS. The variance of the random gain $H_{k q, b m}$ for the $q^{t h}$ antenna of the $k^{t h}$ MS and the $m^{t h}$ antenna of the $b^{\text {th }} \mathrm{BS}$ is set to $E\left[\left|H_{k q, b m}\right|^{2}\right]=1$, and does not reflect realistic system parameters such as shadowing or 


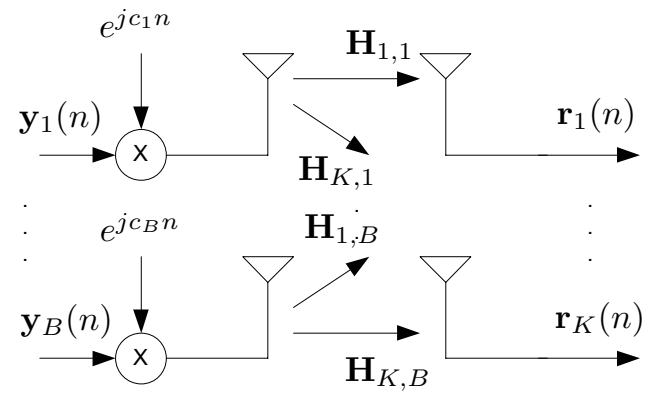

Fig. 1. Coordinated multibase MIMO downlink model

any particular cellular structure. The communications system is considered to be a homogenous cloud of BSs and MSs.

For this work, the zero-forcing downlink beamformer was used. However, other downlink techniques could be substituted. The transmitted signal is constructed for the $k^{t h}$ user by using the nullspace of all other users. Defining $\mathbf{H}=$ $\operatorname{stack}\left(\mathbf{H}_{1}, \mathbf{H}_{2}, \cdots, \mathbf{H}_{K}\right)$, the $(K-1) N_{R} \times B N_{T}$ matrix $\mathbf{H}_{k}^{(-)}$ is $\mathbf{H}$ with all rows from user $k$ removed and the unitary $B N_{T} \times\left(B N_{T}-(K-1) N_{R}\right)$ matrix $\boldsymbol{\Phi}_{k}$ has columns spanning the nullspace of $\mathbf{H}_{k}^{(-)}$. We will refer to $\boldsymbol{\Phi}_{k}$ as the transmit filter. The transmitted signal is represented in the length- $B N_{T}$ vector $\mathbf{x}_{k}(n)=\mathbf{\Phi}_{k} \mathbf{s}_{k}(n)$, with the length- $\left(B N_{T}-(K-1) N_{R}\right)$ vector $\mathbf{s}_{k}(n)$ containing the transmit power and information symbols for MS $k$. The length- $N_{T}$ vector $\mathbf{y}_{b}(n)$ contains the transmit signal for the $b^{t h} \mathrm{BS}$, where $\left[\mathbf{y}_{1}^{T}(n), \cdots, \mathbf{y}_{B}^{T}(n)\right]^{T}=$ $\sum_{k=1}^{K} \mathbf{x}_{k}(n)$. The variance of each component of the noise vector $\mathbf{n}_{k}(n)$ is $N_{0}$.

\section{B. Carrier Frequency Offset}

In the absence of any CFO, (1) is free of inter-user or intersymbol interference. With the introduction of an independent $\mathrm{CFO}$ at each BS, each signal arriving at a MS will be shifted in frequency to a different degree. The resulting progressive phase rotations can be seen as a perturbation on the channel state that degrades the orthogonality of the transmit and receive filters.

The BS CFO matrix $\mathbf{D}(\mathbf{c} n)$ represents the rotation of the symbols due to the CFO at each BS LO, where

$$
\mathbf{D}(\mathbf{c} n)=\operatorname{blkdiag}\left(\mathbf{D}_{1}\left(c_{1} n\right), \cdots, \mathbf{D}_{B}\left(c_{B} n\right)\right) \text {. }
$$

The $b^{\text {th }}$ component matrix is $\mathbf{D}_{b}\left(c_{b} n\right)=e^{j c_{b} n} \mathbf{I}_{N_{T}}$, where $c_{b}=2 \pi f_{b} T_{s}$ is the normalized CFO with a CFO of $f_{b}=\varepsilon_{0} f_{c}$ $\mathrm{Hz}$, a carrier frequency of $f_{c} \mathrm{~Hz}$, and a symbol period of $T_{s}$ seconds.

Since the signal arriving at each MS is a combination of the BS transmissions, there is no method by which the MS can restore the degraded ZF nulls. Each MS must estimate the $\mathrm{CFO}$ for each BS and transmit this information back for future compensation at the BSs. We will present algorithms for the correction method in a future paper.

\section{CFO EFFECTS ON SUM-RATE CAPACITY}

Next, we examine the effects of $\mathrm{CFO}$ in a cooperative multibase MIMO (C-MB-MIMO) system using the sum rate capacity. In essence, the CFOs $c_{b}$ induce phase offsets (POs) $p_{b}$ that increase with time. By symbol time $n$, we have $p_{b}=c_{b} n$. The POs degrade the ZF beamformer by weakening the signal strength and by reducing the depth of nulls, thereby increasing interference. In what follows, we parameterize the capacity analysis by the range of POs, implicitly specifying the range of CFOs and/or length of block. The capacity will be found as a function of the channel state and the PO. The length- $B$ vector $\mathbf{p}=\mathbf{c} n$ contains the PO values for the BSs, so we can also define the CFO matrix $D(\mathbf{c} n)$ as $D(\mathbf{p})$.

Some investigation has been done into the capacity of the downlink of a zero-forcing system in [7]. An expression for the capacity of each user can be derived by using the equation from the appendix of [8]. For the $k^{t h}$ user,

$$
C(\mathbf{H}, \mathbf{p})_{k}=\log _{2} \frac{\left|E\left[\mathbf{x}_{k} \mathbf{x}_{k}^{\dagger}\right]\right|\left|E\left[\mathbf{r}_{k} \mathbf{r}_{k}^{\dagger}\right]\right|}{\left|E\left[\mathbf{u}_{k} \mathbf{u}_{k}^{\dagger}\right]\right|},
$$

where $\mathbf{u}_{k}=\left[\begin{array}{ll}\mathbf{x}_{k}^{T} & \mathbf{r}_{k}^{T}\end{array}\right]^{T}$. The resulting sum-rate capacity for the C-MB-MIMO system is

$$
\begin{aligned}
& C(\mathbf{H}, \mathbf{p})=\sum_{k=1}^{K} \log _{2} \mid \mathbf{I}_{N_{R}}+ \\
& \tilde{\mathbf{H}}_{k} \mathbf{X}_{k} \tilde{\mathbf{H}}_{k}^{\dagger}\left(\sum_{i \neq k}^{K} \tilde{\mathbf{H}}_{k} \mathbf{X}_{i} \tilde{\mathbf{H}}_{k}^{\dagger}+\mathbf{I}_{N_{R}}\right)^{-1} \mid,
\end{aligned}
$$

where $\tilde{\mathbf{H}}_{k}=\mathbf{H}_{k} \mathbf{D}(\mathbf{p}), \quad \mathbf{X}_{k}=\gamma \boldsymbol{\Phi}_{k} \boldsymbol{\Phi}_{k}^{\dagger}, \quad \gamma=$ $E_{B S} /\left(N_{0} K\left(B N_{T}-(K-1) N_{R}\right)\right)$ is the transmit SNR per symbol. Note that the total system energy $E_{B S}$ is used to demonstrate the effect of the number of independent LOs on the sum-rate capacity while maintaining a constant transmit energy. In (4) we are assuming equal power transmission $\left(E\left[\mathbf{s}_{k}(n) \mathbf{s}_{k}^{\dagger}(n)\right]=\gamma \mathbf{I}\right)$, without waterfilling. The terms within the inverse are considered to be the effective noise, and consist of the interference plus noise covariance. If the CFOs were zero, so that $\mathbf{D}(\mathbf{p})=\mathbf{I}_{B N_{T}}$, then the interference term would disappear due to the orthogonality of the $K$ transmit filters. Consequently, $C(\mathbf{H}, \mathbf{0})=\sum_{k=1}^{K} \log _{2}\left|\mathbf{H}_{k} \mathbf{X}_{k} \mathbf{H}_{k}^{\dagger}+\mathbf{I}_{N_{R}}\right|$.

To find the average capacity, the expectation of (4) over $\mathbf{H}$ and $\mathbf{p}$ was taken with 10,000 Monte Carlo simulations. The channel state matrix components were set to zero mean, unit variance, complex Gaussian random variables. A maximum PO $p_{\max }$ was used to determine the effect of absolute phase offset on the sum rate capacity. We used this on (4), where $\mathbf{D}(\mathbf{p})=\operatorname{blkdiag}\left(e^{j p_{\max } \theta_{1}} \mathbf{I}_{N_{T}} \cdots e^{j p_{\max } \theta_{B}} \mathbf{I}_{N_{T}}\right)$ and $\theta_{b}$ is a uniform random variable with a range of $[-1,+1]$. $p_{\max }$ could be replaced with $c_{\max } n$ if parameterization on symbol time $n T_{s}$ were desired.

Fig. 2 shows the effect of CFO on capacity for a total transmit SNR of $E_{B S} / N_{0}=20 \mathrm{~dB}$, with the power divided evenly between the $K$ users among the $B N_{T}$ antennas. The fixed system parameters are $K=32$ and $N_{R}=1$. For all curves, the degrees of freedom $B N_{T}-(K-1) N_{R}=1$, so we have the zero-forcing system with one stream per user (i.e. unit 


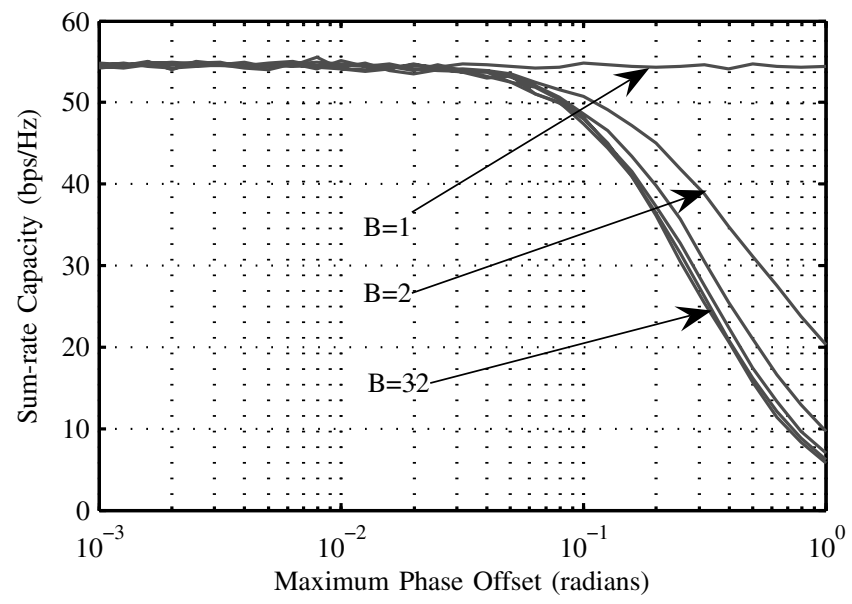

Fig. 2. Effect of CFO on sum-rate capacity; $E_{B S} / N_{0}=20 \mathrm{~dB}$ total transmit SNR; $B=1,2,4,8,16,32 ; K=32 ; N_{R}=1 ; B N_{T}=32$

rank gain matrices $\mathbf{H}_{k}$ ). The total number of transmit antennas $B N_{T}$ was kept constant at 32 , so the curves demonstrate the effect of independent LOs. The capacity drops as the number of independent LOs, $B$, increases. This demonstrates the effect of the random phase offset. The loss of capacity as the PO increases can be viewed as the cumulative effect of the CFO over time. Given an initially accurate channel estimate, which subsumes the CFO phase error at the moment of the estimate, the orthogonality between the transmitted streams degrades as time increases. For example, for the $B=32$ system, the capacity drops by about $20 \%$ when the PO is 0.1 radians. For a 500 symbol frame, $f_{c}=2.4 \mathrm{GHz}$ and $T_{s}=1 \mu \mathrm{s}$, this means a maximum allowable $\mathrm{CFO}$ of $0.08 \mathrm{ppm}$. For many communication systems, this is a very optimistic value. If we were also to include CSI feedback delay, it is important to consider that the system will start at a slightly reduced capacity level before transmitting even a single data bit.

We next compared the effects of LO CFO to Doppler shift on sum-rate capacity. Since both phenomena involve a frequency shift in the modulated carrier, we use the ratio $R=f_{b} / f_{b, D} \approx \varepsilon_{0} / 10^{-9} s_{b}$ to compare the severity of the CFOs. $f_{b, D}$ is the Doppler frequency and $s_{b}$ is the relative speed of the MS in $\mathrm{km} / \mathrm{h}$, both referenced to the $b^{t h}$ BS. For a vehicular speed of $50 \mathrm{~km} / \mathrm{h}$ and a LO CFO accuracy of $\varepsilon_{0}=1$ ppm (i.e. $10^{-6}$ ), $R=20$. In other words, the effect of $\mathrm{LO}$ CFO is 20 times greater than Doppler shift, or equivalent to a system with a LO CFO of $0.05 \mathrm{ppm}$. Note that this comparison is independent of carrier frequency.

Another cause of time-dependent capacity degradation is channel fading. To incorporate channel fading into our capacity model, we used the Jakes fading model with Doppler frequency $f_{b, D}$ for the channel state autocorrelation. Assuming that all BSs have the same Doppler frequency $f_{b, D}=f_{D}$, the capacity expression (4) can be rewritten with $\tilde{\mathbf{H}}_{k, D}=$ $\rho(n) \mathbf{H}_{k}+\sqrt{1-\rho^{2}(n)} \boldsymbol{\Upsilon}_{k}$, where $\rho(n)=J_{0}\left(2 \pi n f_{D} T_{s}\right)$, $J_{0}(x)$ is the zeroth order Bessel function and the channel

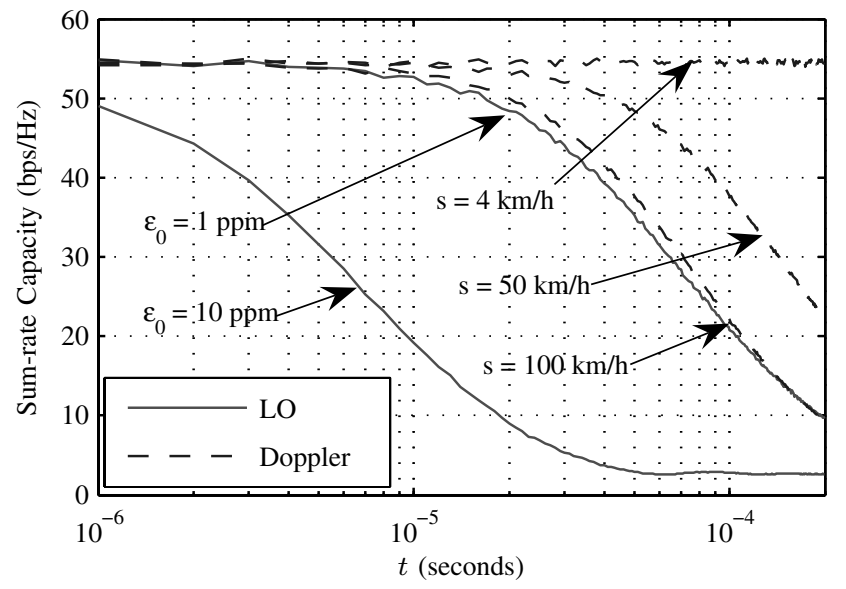

Fig. 3. Sum-rate capacity average versus time for CFO due to local oscillator offset and Doppler spread; $E_{B S} / N_{0}=20 \mathrm{~dB}$ total transmit SNR; $B=4$; $N_{T}=8 ; K=32 ; N_{R}=1 ; T_{s}=1 \mu \mathrm{s} ; f_{c}=5 \mathrm{GHz}$

correlation of the Jakes Doppler model, and $\Upsilon_{k}$ is a $B N_{T} \times$ $N_{R}$ matrix of complex Gaussian random variables with zero mean and unit power. The curves in Fig. 3 demonstrate that the effect of LO offset on capacity is more severe than Doppler spread for normal system parameters. For a carrier frequency of $f_{c}=5 \mathrm{GHz}$ and a symbol period of $T_{s}=1 \mu \mathrm{s}$, capacity curves are shown for a variety of Doppler spreads. Curves for LO offsets of 1 and $10 \mathrm{ppm}$ are shown for comparison. Note that the LO offset curves are averaged over receiver noise $\mathbf{n}_{k}$ and the offsets $\mathbf{c}$, which is modeled as a uniformly distributed random variable, while the Doppler spread curves are averaged over receiver noise $\mathbf{n}_{k}$ and channel noise $\boldsymbol{\Upsilon}_{k}$. The implication is that the Doppler curves are for a worst case $f_{D}$, while the LO CFO curves are not, and so the results are. For the $10 \mathrm{ppm}$ LO CFO curve, the sum-rate capacity is at $36 \%$ full capacity by the time the Doppler spread even begins to show any effect, even at the high speed of $100 \mathrm{~km} / \mathrm{h}$.

\section{CFO ESTIMATION}

\section{A. Limits on Sequence Length}

For channel and CFO estimation, training sequences (TS) of length $N$ are used. For MIMO systems, the optimum TS set consists of orthogonal sequences with a length greater than or equal to the number of transmit antennas $\left(N \geq B N_{T}\right)$ [9]. However, the MSE of the CSI is inversely dependent on both the length of the sequence and its energy (the derivation is not presented due to space constraints). Since the transmit power is normally limited due to amplifier or spectral mask constraints, the TS length will generally need to be much greater than the number of transmit antennas in order to achieve a useful MSE level, which in turn will determine an achievable sum rate capacity. However, the longer the TS, the lower the data throughput. The selection of $N$ is thus a trade-off between instantaneous capacity and data throughput.

The CFO estimate will contain residual error. This residual $\mathrm{CFO}$, or the estimator error variance, may depend on the 
sequence length, transmit power, and the number of BSs, and is especially of concern if a MSE floor exists for the estimator. Considerations such as this are important for the system designer.

\section{B. Existing Joint CSI and CFO Estimators}

Although the estimation of single frequency offsets is a classical and well-understood problem, there has been little work on the estimation of multiple CFOs from interfering data signals or training sequences (TSs). In [4], Besson and Stoica develop a solution using ML estimation of the CFOs based on an LS solution for the CSI. The synchronization sequences are forced into orthogonality by restricting each BS to a specific time slot. This prevents any TS correlation. We will refer to this technique as TO for "time orthogonalization". In [5], Ahmed et al. exploit the fact that in certain cases, the code covariance matrix off-diagonals will be very small compared to the diagonals. This allows each CFO to be estimated independently from one another. We will refer to this technique as ST-AO for "simultaneous training - assumed orthogonalization". The rationale in both cases is that accounting for the correlation is computationally intensive. For our C-MB-MIMO model, we have multiple transmit and receive antennas with the same CFO, so the ST-AO and TO algorithms have been slightly modified to take advantage of this.

\section{Proposed Gradient Technique}

The previous techniques are inappropriate for the coordinated downlink. In TO, the training sequences do not overlap in time. Consequently, the length of the composite TS increases linearly as the number of BSs $B$ increases, without providing any corresponding increase in accuracy of the resulting frequency estimates. In ST-AO, the effect of sequence correlation is ignored; however, if the TS are too long, the approximation is invalid.

In our proposed method, we transmit all TSs simultaneously as in ST-AO. In contrast to ST-AO, we account for the TS correlation introduced by the CFOs. The trade-off between the use of simultaneous TSs versus time orthogonal TSs is between sequence length and symbol power. For example, to ensure equal TS energy with $B \mathrm{BSs}$, a time orthogonal system would have to use TSs that are $B$ times less in duration with a per-symbol power $B$ times higher than with simultaneous training. This trade-off favours simultaneous training, given that power amplifiers are usually one of the most expensive parts of a BS. Simultaneous training has the problem that TS orthogonality is degraded by the CFOs. We solve that problem below with a gradient-based estimator, which we term ST-G, for "simultaneous training - gradient."

Denote $\mathbf{X}=\left[\mathbf{X}_{1} \cdots \mathbf{X}_{B}\right]$ as an $N \times B N_{T}$ matrix of length$N$ synchronization sequences, where the $N \times N_{T}$ sub-matrix $\mathbf{X}_{b}$ denotes the sequences for the $b^{t h} \mathrm{BS}$. The TS as seen by the MS is

$$
\begin{aligned}
\mathbf{V}(\mathbf{c}) & =\left[\mathbf{X}_{1} \odot \boldsymbol{\Omega}_{1}\left(c_{1}\right) \cdots \mathbf{X}_{B} \odot \boldsymbol{\Omega}_{B}\left(c_{B}\right)\right] \\
& =\mathbf{X} \odot \boldsymbol{\Omega}(\mathbf{c}),
\end{aligned}
$$

which denotes the element-by-element product of the synchronization sequences and a matrix of CFO-induced symbol rotations. The $N \times N_{T} \Omega_{b}\left(c_{b}\right)$ matrix is constructed with all $N_{T}$ columns equal to the same length- $N$ column vector $\boldsymbol{\omega}_{b}\left(c_{b}\right)=\left[1 e^{j c_{b}} \cdots e^{j c_{b}(N-1)}\right]^{T}$.

The received samples for the $k^{t h}$ MS can be represented in a length- $N N_{R}$ vector

$$
\mathbf{r}_{k}=\overline{\mathbf{V}}(\mathbf{c}) \mathbf{h}_{k}+\mathbf{n}_{k}
$$

where $\overline{\mathbf{V}}(\mathbf{c})=\mathbf{I}_{N_{R}} \otimes \mathbf{V}(\mathbf{c})$ refers to the block diagonalization of $N_{R}$ copies of $\mathbf{V}(\mathbf{c})$ with $\otimes$ being the Kronecker product, $\mathbf{h}_{k}$ is the length- $N_{T} N_{R}$ vector of channel gains created by stacking the columns of $\mathbf{H}_{k}$, and $\mathbf{n}_{k}$ is the length- $N N_{R}$ vector of additive white gaussian noise.

As demonstrated in [4] and [5], the optimum ML estimator of the length- $B$ vector $\mathbf{c}$ is

$$
\hat{\mathbf{c}}=\underset{\mathbf{c}}{\operatorname{argmax}} \mathbf{r}_{k}^{\dagger} \overline{\mathbf{V}}(\mathbf{c})\left(\overline{\mathbf{V}}(\mathbf{c})^{\dagger} \overline{\mathbf{V}}(\mathbf{c})\right)^{-1} \overline{\mathbf{V}}(\mathbf{c})^{\dagger} \mathbf{r}_{k}
$$

where the CSI estimate is $\hat{\mathbf{h}}_{k}=\left(\overline{\mathbf{V}}(\hat{\mathbf{c}})^{\dagger} \overline{\mathbf{V}}(\hat{\mathbf{c}})\right)^{-1} \overline{\mathbf{V}}(\hat{\mathbf{c}})^{\dagger} \mathbf{r}_{k}$. This optimization is $B$-dimensional, involving a $B N_{T} \times B N_{T}$ matrix inverse (by exploiting the block diagonal nature of $\overline{\mathbf{V}}(\mathbf{c})$ ). Provided the TS length $N$ is short enough to prevent any aliasing ("sidelobes") within the search domain, the objective function in (7) is convex, as is the search domain. Aliasing is absent if $N<\pi / 2 c_{\max }$, where the factor of $1 / 2$ is from a lower approximation to the inflection point of the $\operatorname{sinc}(x)$ windowing function in the interval $[0,1]$ and $c_{\max }$ refers to the maximum normalized CFO. In this case, we can use a gradient technique to search for the zero-gradient point [10]. Due to space limitations, a detailed investigation into the convexity restrictions on $N$ and $c_{\max }$ will be done in an upcoming journal paper; for now, we only consider $N$ and $c_{\max }$ that meet the above constraint. Defining $\Lambda(\mathbf{c})=$ $\mathbf{r}_{k}^{\dagger} \overline{\mathbf{V}}(\mathbf{c})\left(\overline{\mathbf{V}}(\mathbf{c})^{\dagger} \overline{\mathbf{V}}(\mathbf{c})\right)^{-1} \overline{\mathbf{V}}(\mathbf{c})^{\dagger} \mathbf{r}_{k}, N_{\text {ITER }}$ as the number of iterations, and $t$ as the step size, a basic algorithm is

$$
\begin{aligned}
& \text { initialize } \hat{\mathbf{c}}_{0}=\mathbf{0} \\
& \text { for } i=1: N_{I T E R} \\
& \quad \hat{\mathbf{c}}_{i}=\hat{\mathbf{c}}_{i-1}-t \nabla \Lambda\left(\hat{\mathbf{c}}_{i-1}\right) \\
& \text { end. }
\end{aligned}
$$

We can find the gradient of $\Lambda(\mathbf{c})$ with a combination of analytical and numerical evaluation. The $b^{\text {th }}$ component of the length- $B$ vector $\nabla \Lambda(\hat{\mathbf{c}})$ can be expressed as:

$$
\frac{\partial}{\partial c_{b}} \Lambda(\hat{\mathbf{c}})=\mathbf{r}_{k}^{\dagger}\left[\overline{\mathbf{G}}_{b}(\hat{\mathbf{c}})\right] \mathbf{r}_{k}
$$

where

$$
\begin{aligned}
\mathbf{G}_{b}(\hat{\mathbf{c}})= & \mathbf{A}_{b}\left(\hat{c}_{b}\right) \mathbf{F}(\hat{\mathbf{c}}) \mathbf{V}^{\dagger}(\hat{\mathbf{c}})+\mathbf{V}(\hat{\mathbf{c}}) \mathbf{F}(\hat{\mathbf{c}}) \mathbf{A}_{b}^{\dagger}\left(\hat{c}_{b}\right) \\
& -\mathbf{V}(\hat{\mathbf{c}}) \mathbf{B}_{b}(\hat{\mathbf{c}}) \mathbf{F}(\hat{\mathbf{c}}) \mathbf{B}_{b}(\hat{\mathbf{c}}) \mathbf{V}^{\dagger}(\hat{\mathbf{c}}) .
\end{aligned}
$$

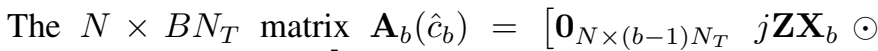
$\left.\boldsymbol{\Omega}_{b}\left(\hat{c}_{b}\right) \quad \mathbf{0}_{N \times(B-b) N_{T}}\right]$, the $N \times M$ matrix $\mathbf{0}_{N \times M}$ is an all zero matrix, the $B N_{T} \times B N_{T}$ matrix $\mathbf{B}_{b}(\hat{\mathbf{c}})=$ 
$\mathbf{A}_{b}^{\dagger}\left(\hat{c}_{b}\right) \mathbf{V}(\hat{\mathbf{c}})+\mathbf{V}^{\dagger}(\hat{\mathbf{c}}) \mathbf{A}_{b}\left(\hat{c}_{b}\right)$, the $B N_{T} \times B N_{T}$ matrix $\mathbf{F}(\hat{\mathbf{c}})=\left(\mathbf{V}^{\dagger}(\hat{\mathbf{c}}) \mathbf{V}(\hat{\mathbf{c}})\right)^{-1}$, and the $N \times N$ matrix $\mathbf{Z}=$ $\operatorname{diag}(0,1,2 \cdots N-1)$.

The convergence of the algorithm in (8) is controlled by the step size $t$. The optimized $t$ is defined as the value that allows convergence in the fewest iterations. Since the gradient step in (9) is dependent on the channel state from $\mathbf{r}_{k}$, the optimized value of $t$ fluctuates due to channel fades and may be different for each BS (i.e. each CFO). A method to account for this is to allow each CFO gradient component its own step size. This can be done with the assumption that the CFO estimates are uncorrelated (verified by simulation of $\Lambda(\hat{\mathbf{c}})$ ). The new CFO-specific weights, $t_{b}$ for the $b^{t h} \mathrm{CFO}$, include the term $\left\|\mathbf{H}_{k, b}\right\|_{F}$, where $\|\cdot\|_{F}$ refers to the Frobenius norm of the zero-mean channel gain matrix. Since the channel gains are not available, an estimate must be calculated. Simulations show that the estimate

$$
\hat{\mathbf{H}}_{k, b}=\left(\mathbf{X}_{b}^{\dagger} \mathbf{X}_{b}\right)^{-1} \mathbf{X}_{b}^{\dagger} \mathbf{r}_{k}
$$

is satisfactory, even in the presence of CFO. This estimate is already available from the channel estimation process, which proceeds in parallel with CFO estimation. The improved update to the CFO estimate in (8) now becomes

$$
\hat{\mathbf{c}}_{i}=\hat{\mathbf{c}}_{i-1}-\mathbf{T} \nabla \Lambda\left(\hat{\mathbf{c}}_{i-1}\right),
$$

where $\mathbf{T}$ is a $B \times B$ diagonal matrix with diagonal components $t_{b}=a /\left\|\hat{\mathbf{H}}_{k, b}\right\|_{F}$ and $a$ is a constant.

To accommodate $\mathrm{CFO}$ magnitudes that are greater than $c_{\max }=\pi / 2 N$, the TS can be segmented such that each individual segment satisfies the convexity constraint. The segments are combined by non-coherent addition of (9) for each segment. The details of this more robust algorithm will be submitted separately.

\section{Simulations}

We compared the TO, ST-AO and ST-G estimators. The TO and ST-AO estimators were modified to fit our C-MB-MIMO model. Both the ST-AO and TO rely upon a search over a range of CFO values, consisting of $N$ frequency bins, suitable for the FFT. Monte Carlo simulations of 1000 runs were used with a Rayleigh distributed channel state. The channel state was static for the duration of the TS. The CFO is random and uniformly distributed, with $c_{b} \in\left[-c_{\max },+c_{\max }\right]$ and $c_{\max }=0.01$. The number of BS was set to $B=4$, and for ST-AO and ST-G, $N=128$. For TO, the TS length per BS was reduced by $B$ to $N=32$; since TO TSs require interleaving by symbol [4], each BS will transmit one TS symbol every $B$ symbols. This interleaving would be difficult to do in practice, but it allows the TO technique to maintain the same aperture as the full length ST-AO and ST-G sequences. Thus, symbol synchronous transmission is assumed for these simulations, although a buffer period after each symbol could serve the same purpose. For ST-G however, the metric could be expanded to include symbol timing estimation. This will be covered in a future publication.

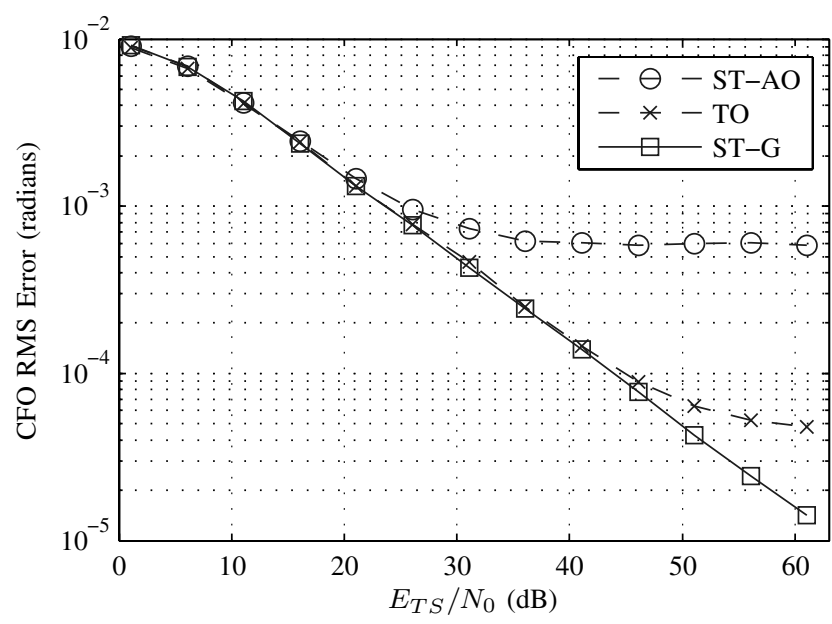

Fig. 4. Comparison of estimation techniques over SNR; $B=4, N_{T}=$ $2, N_{R}=2, c_{\max }=0.01, N=128, N_{I T E R}=20, t=1 \times$ $10^{-6} N_{0} /\left\|\hat{\mathbf{H}}_{k, b}\right\|_{F} E_{S}$; NOTE: Peak power of TO is $B$ times that of ST-G and ST-AO.

To keep the comparison between the techniques fair, energy comparisons used the TS energy over the noise, $E_{T S} / N_{0}$. With a total transmit energy $E_{S}$ allocated to each BS, the transmit energy per symbol is $E_{S} / N_{T}$, resulting in $E_{T S} / N_{0}=$ $N E_{S} / N_{T} N_{0}$ for both ST-G and ST-AO. Since the TO sequence lengths are reduced by $B$, the symbol energy must be increased by $B$ to keep the same $E_{T S} / N_{0}$ value. It is very important to note that while this is a fair comparison on the basis of transmitted energy levels, the radio power amplifiers used for the TO technique are required to handle $B$ times more power than in either the ST-AO or ST-G techniques. The remaining parameters were set to $N_{T}=2$ and $N_{R}=2$.

Fig. 4 compares the estimator root mean square (RMS) error versus signal energy, Fig. 5 compares the estimator RMS error versus TS length, and Fig. 6 compares the estimator RMS error versus the number of BSs. For TO, $N$ is corrected for all graphs as explained in the previous paragraph. The symbol SNR of $E_{S} / N_{0}=30 \mathrm{~dB}$ in Fig. 5 and Fig. 6 corresponds to a TS SNR of $E_{T S} / N_{0}=51.1 \mathrm{~dB}$ in Fig. 4. Also note that the curve for ST-G in Fig. 5 does not exist above $N=128$. This is a result of the convexity constraint in Section IV-C.

The effect of sequence correlation in the ST-AO technique due to accumulated phase rotations is evident in the error floor of Fig. 4. The TO technique does not exhibit this error floor since the sequences are forced to be orthogonal. The ST-G technique also avoids this error floor since it is guaranteed to converge due to the convexity of $\Lambda(\mathbf{c})$. For both ST-AO and TO, an error floor due to finite FFT bin width may also occur. This is visible for TO in Fig. 4 as an incipient floor in RMS error at high $E_{T S} / N_{0}$. ST-G avoids having to use a discrete search, and thus avoids this error floor as well.

The SNR in Fig. 5 and Fig. 6 is chosen such that ST-AO is operating with an error floor. In Fig. 5, the decrease of CFO RMS error is algebraic in $N$; with the parameters specified, a decrease of the CFO RMS error by a factor of 10 requires an 


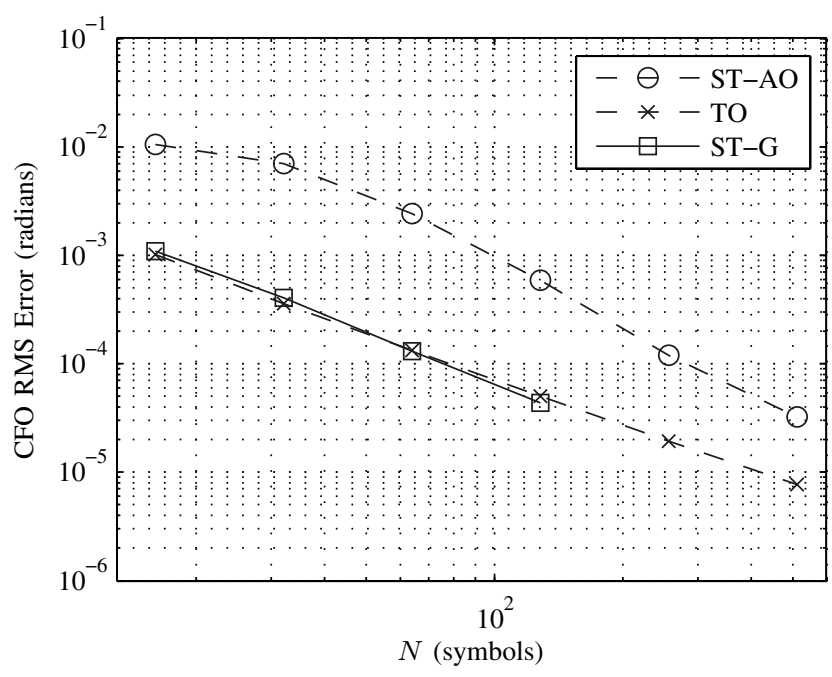

Fig. 5. Comparison of estimation techniques over TS length; $B=4, N_{T}=$ $2, N_{R}=2, c_{\max }=0.01, E_{S} / N_{0}=30 \mathrm{~dB}, N_{I T E R}=20$; NOTE: Peak power of TO is $B$ times that of ST-G and ST-AO.

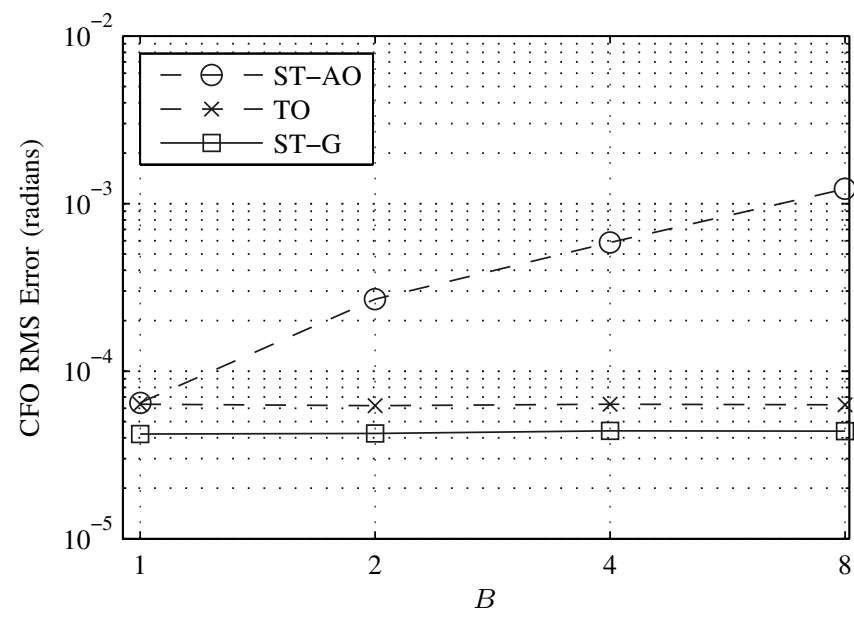

Fig. 6. Comparison of estimation techniques over number of BSs; $N_{T}=2$, $N_{R}=2, c_{\max }=0.01, E_{S} / N_{0}=30 \mathrm{~dB}, N=128, N_{I T E R}=20$; NOTE: Peak power of TO is $B$ times that of ST-G and ST-AO.

increase in the TS length $N$ by a factor of approximately 5.2 . Also note that the performance of ST-G and TO match until the TS length violates the convexity constraint. For Fig. 6, the curve for TO and ST-G is flat for the values of B chosen, but ST-AO shows variation. Since the SNR is high enough to be in the error floor region for ST-AO, adding additional BSs is equivalent to adding extra interferers. For both ST-G and TO, additional BSs do not alter CFO RMS error to a noticeable degree. The gap between ST-G and TO is caused by the bin size of the TO FFT.

The number of iterations of ST-G necessary for convergence was determined with simulation. The maximum number of iterations, $N_{I T E R}$, was set to $N_{I T E R}=20$ for the simulations in Fig. 4, Fig. 5, and Fig. 6. To reduce computations, once a normalized CFO estimate has converged sufficiently, no more iterations are performed for that specific CFO. This can be done since the CFO estimates are uncorrelated (Section IV-C). For these simulations, once the normalized CFO is changing by less than $0.1 \%$ between iterations, it is removed from the set of CFOs. This allows effective convergence in an average of 8 iterations. The $b^{\text {th }}$ CFO step size constant from (12) was set to $a=1 \times 10^{-6} N_{0} / E_{S}$ for $N=128$. It is important to note that $t$ is also dependent on the system dimensionality.

For computational complexity, ST-G requires approximately $B N^{2} N_{I T E R}\left(2 B N_{T}+N_{R}^{2}\right)$ multiplications. Note that since we are removing individual CFOs after they have converged, $N_{I T E R}$ can be replaced with a mean value. The ST-AO and TO techniques require approximately $N N_{T} N_{R} \log _{2}(N / B)+$ $N^{2} / B$ multiplications, stemming from the length- $N$ FFTs and the sort algorithm.

\section{CONCLUSIONS}

As could be expected, coordinating the complex weights of the antennas of several base stations so that they act as a single large beamformer presents many challenges. We have identified one - the carrier frequency offsets due to independent oscillators at the bases - and quantified the resulting capacity losses. For example, using typical values, even the stringent specification of $0.1 \mathrm{ppm}$ on oscillator stability results in a $20 \%$ loss of capacity over a 500-symbol frame. We also presented a new algorithm by which the mobile can estimate all the base station frequency offsets from mutually interfering training sequences, and provide better accuracy and lower peak power requirements than the few previous investigations of multiple frequency offset estimation.

\section{REFERENCES}

[1] S. V. Hanly, "Capacity and power control in spread spectrum macrodiversity radio networks," IEEE Trans. Commun., vol. 44, no. 2, pp. 247-256, 1996.

[2] L. Welburn, J. K. Cavers, and K. W. Sowerby, "Multiuser-macrodiversity detection in rayleigh fading channels," in IEEE Veh. Tech. Conf., vol. 5 , 2000, pp. 2340-2347.

[3] M. N. Bacha, J. S. Evans, and S. V. Hanly, "On the capacity of mimo cellular networks with macrodiversity," in IEEE Australian Commun. Theory Workshop, vol. 1, 2006, pp. 105-109.

[4] O. Besson and P. Stoica, "On parameter estimation of mimo flat-fading channels with frequency offsets," IEEE Trans. Signal Process., vol. 51, no. 3, pp. 602-613, 2003.

[5] S. Ahmed, S. Lambotharan, A. Jakobsson, and J. A. Chambers, "Mimo frequency-selective channels with multiple-frequency offsets: estimation and detection techniques," IEE Proc. Commun., vol. 152, no. 4, pp. 489494, 2005

[6] M. K. Oh, X. Ma, G. B. Giannakis, and D. J. Park, "Cooperative synchronization and channel estimation in wireless sensor networks," in Conf. Record of Thirty-Seventh Asilomar Conf. On Signals, Systems and Computers, vol. 1, 2003, pp. 238-242.

[7] T. Yoo and A. Goldsmith, "On the optimality of multiantenna broadcast scheduling using zero-forcing beamforming," IEEE J. Sel. Areas Commun., vol. 24, no. 3, pp. 528-541, 2006.

[8] G. J. Foschini and M. J. Gans, "On limits of wireless communications in a fading environment when using multiple antennas," Wireless Personal Commun., vol. 6, no. 3, pp. 311-335, 1998.

[9] M. Biguesh and A. B. Gershman, "Mimo channel estimation: optimal training and tradeoffs between estimation techniques," in IEEE Int. Conf. on Commun., vol. 5, 2004, pp. 2658-2662.

[10] S. Boyd and L. Vandenberghe, Convex Optimization. Cambridge, UK: Cambridge University Press, 2006. 